\title{
Theorising Digital Tools: Mutual Constitution of the Person and Digital in a Conceptual PlayWorld
}

\author{
Prabhat Rai $^{1}$ (D) Marilyn Fleer ${ }^{2}$ (D) . Glykeria Fragkiadaki ${ }^{1}$ (D)
}

Received: 24 July 2020 / Revised: 25 October 2020 / Accepted: 11 December 2020 /

Published online: 11 January 2021

(c) The Author(s), under exclusive licence to Springer Nature Switzerland AG part of Springer Nature 2021

\begin{abstract}
The COVID-19 pandemic has led us to an unanticipated and challenging world. In educational settings, one of the ways to respond to this crisis has been the online delivery of content. One of the valid critiques of these online delivery models is the passive role of the participants. The challenge is we still know very little about how technology could support children's active participation in their learning. This challenge exacerbates with children in early years and in their home setting, which is the focus of our research at Monash University's Conceptual PlayLab. This paper reports on solving the theoretical problem of digital design that can create a relational space for children's STEM concept formation. The paper argues that engagement with digital tools demands clear design principles that could support both robust concept formation and also development of learning motives in children. Drawing on Wartofsky's work on artefacts and tool-use and (Fleer 2017a, 2019), conceptualisation of "digital coadjuvant", we make a case that technology offers a transformative potential for children to imagine and explore together with their caregivers in the Conceptual PlayWorld. We have showed through a practice example on how person and digital technologies become mutually constituted in a pop-up Conceptual PlayWorld. The design principle highlighted in the paper argues for transcending the binary of digital and non-digital. The paper concludes that Conceptual PlayWorld offers a design model that uses digital tools to sustain and amplify children's exploration in their imaginary play situation.
\end{abstract}

Keywords Conceptual PlayWorld · Digital tools · Spatial concept learning · Models · Motives

Prabhat Rai

prabhat.rai@monash.edu

Marilyn Fleer

Marilyn.Fleer@monash.edu

Glykeria Fragkiadaki

Glykeria.Fragkiadaki@monash.edu

1 Senior Research Fellow, Conceptual PlayLab, Monash University, Peninsula Campus, Melbourne, VIC 3199, Australia

2 Laureate Professor, Conceptual PlayLab, Monash University, Peninsula Campus, Melbourne, VIC 3199, Australia 


\section{Introduction}

Digital technology has come to be seen as one of our saviours in the post-COVID world. It seems to have either sustained our previous modes of interaction or in some cases created possibilities for reimagining new ways of engaging with the world. The enormous possibilities appear to be located within a context of passive consuming of a technological world. Alerting us to this pliant engagement with technology, Derry (2007) argues that "the potential of technology to enhance learning can only be realized if it is based on a secure foundation of a robust understanding of learning, teaching and knowledge" (Derry 2007, p.503). In the context of digital technology, the "design/architecture of learning" is of utmost importance. Yet, few have studied how person and digital technologies become mutually constituted through design. This paper takes up this challenge by reporting on emerging research into a pop-up Conceptual PlayWorld designed as part of a suite of studies being undertaken through the Conceptual PlayLab at Monash University (https:// www.monash.edu/conceptual-playworld). The outcomes of the study make a twofold claim: one to highlight the role of a Conceptual PlayWorld as a "model" that creates motivating conditions for children's STEM concept learning in home settings and challenges the binary of digital and non-digital, and two, to foreground the relational nature of the use of digital tools in children's home setting.

\section{Mutual Constitution of the Individual and Digital}

The research tradition espoused by Vygotsky through his cultural-historical approach and philosophical work of Wartofsky's on historical epistemology has been used to develop a holistic understanding of the practices related to digital tools. The focus on sociogenesis, i.e. social foundations of human practice and hence development of psychological functions, demands that instead of focusing merely on "digital tool", a holistic approach that looks at the "design of digital tool-use" is needed. A central tenet for Vygotsky is that we do not act directly upon the world; it is always mediated by means of sign, symbols, or material tools and always in social relations. Tools carry a history of humans' engagement with their world. Tools and their use thus "reflect the particular accumulated insights of communities, it is not possible to use a tool appropriately without understanding the community or culture in which it is used" (Brown et al. 1989, p. 3). This points towards one of the core Vygotskian insights that learning or human consciousness "is fundamentally a mediated mental activity" (Lantolf and Appel 1994, p. 7).

The longstanding theorising of Wertsch (2007) that is worthy of revisiting. Wertsch explicates the distinction between two perspectives on mediation in Vygotsky's writing: explicit mediation and implicit mediation. Explicit mediation, he explains, involves the intentional and overt introduction of "stimulus means" or signs into an activity. It is explicit also in the sense that the sign or stimulus means is evident to those who are participating in the activity (Wertsch 2007, p. 180). Implicit mediation on the other hand "involves signs in the form of natural language that have evolved in the service of communication and are then harnessed in other forms of activity" (Wertsch 2007, p. 185). In Wertsch's interpretation of Vygotsky, the two kinds of mediation are not in opposition to each other; rather both of them are part of the larger system of "tool-mediated learning actions". What we find missing in Wertsch's work in a contemporary context of digital 
tools is how to capture the motivating conditions which give personal meaning and which are realised through how person and digital technologies become mutually constituted through design.

We turn to Vygotsky's original writings and extend his insights about the relationship between word and thought to understand children's engagement with digital tools. Vygotsky argued that the relationship between word and thought undergo fundamental changes as children learn. He proposed that humans use signs or words before understanding them. As they further engage with them, they gain fuller understanding of those signs. This argument gives us a great insight into how meaning is shared when children engage with digital tools. We are particularly interested in understanding how digital tools amplify or create motivating conditions for children's concept formation. The special importance to the concept of "tools" in humans' understanding of the world and of themselves in Vygotsky's writings hints that similar to other material tools, digital tools are also artefacts created by humans under specific cultural and historical conditions, and thus, they carry the characteristics of the culture. This engagement with digital tools connects us not only with the object but also with people and the practices they inhabit. Seen from these lenses, tools either digital or non-digital are relational in nature.

Similar to Vygotsky, Wartofsky (1979) makes a case for "historical epistemology" and claims that tool use is a distinctive human action. Tool here is referred to as "any artefact created for the purpose of successful production and reproduction of the means of existence" (p.200-201). In this sense, digital tools are central to our social organisation, communication and existence in the present world. They are not mere tools for action but also have commitment reflected in their "normative" conceptualisation. Wartofsky also proposes a related tripartite system of artefacts to explain "historical human praxis". In his system of concepts, primary artefacts are those directly used in production "e.g. axes, clubs, needles, bowls, etc." (p.201), and secondary artefacts are defined as "those used in the preservation and transmission of the acquired skills or modes of action or praxis by which this production is carried out" (p.202). Secondary artefacts are "not in the mind", as mental entities. Wartofsky further explicated that secondary artefacts are

"the products of direct outward action, the transformations of natural materials, or the disposition or arrangement of bodily actions (e.g. in dance) or the social forms of organization of such activities as hunting, or of such relations as kinship, hierarchy, etc. They are externally embodied representations.” (Wartofsky 1979, p. 202).

Tertiary artefact Wartofsky argues are "abstracted from their direct representational function, and suggest that they constitute a domain in which there is a free construction in the imagination of rules and operations different from those adopted for ordinary 'thisworldly' praxis" (p.209). Tertiary artefact creates a "relatively autonomous 'world', in which the rules, conventions and outcomes no longer appear directly practical, or which, indeed, seem to constitute an arena of non-practical, or "free' play or game activity" (p.208). A visible challenge here is the messiness in using these systems of concepts as the same artefact function as primary, secondary or tertiary artefact depending upon the relationship with its user (Gillespie and Zittoun 2010). Cole (2019) critiques Gillespie and Zittoun's position as he endorses "the need to describe one and the same reality from different perspectives" (p.320). This argument relates with Hedegaard's (2008, 2013) emphasis on unpacking the societal needs and institutional practices in which the person navigates meaning and align their motives of participation in an activity setting. It is this collective theorisation that has inspired us to solve the problem of how person and digital technologies become mutually constituted through design. 


\section{Conceptual PlayWorld Design for Digital Engagements in Home Setting}

The study explores Conceptual PlayWorld as a model of planned intervention in children's home setting. Conceptual PlayWorlds is a theoretically informed playbased model that creates motivating conditions for children's concept formation using children's stories. The characteristics of the models are explained further in the next section. Fleer (2017b) has argued that the use of "digital devices... in Playworlds, amplified the children's play in scientific ways, which in turn created new conditions for children's development" (p.303). The digital tools thus support children's meta-position with respect to their play. It transcends their existing relationship with the concrete world. While playing within the Conceptual PlayWorld, young children develop their understandings about the natural, technical, and technological world in relation to their social and cultural reality. The arguments developed in this paper shows that Conceptual PlayWorld as a design model carries with it the societal needs, institutional practices in which the person navigates as part of the process of how person and digital technologies become mutually constituted. These design understandings are critical to lay the foundations for the construction of precursor models in child's thinking that are compatible with the scientific models used in education (Ravanis 2000, 2005) as well as for the formation of complex schemes and advanced mental models (Johnson-Laird 1983).

Drawing on Vygotsky's work Fleer $(2017 \mathrm{a}, \mathrm{b})$ has articulated that a theoretical understanding from a cultural-historical perspective shall consider technology as offering transformative possibilities for children's development. Challenging the binary between digital and non-digital technology, technology can be seen as an auxiliary tool that can open new pathways for children's development. This is a different conceptualisation to that of earlier interpretations of Vygotksy's writings by Wertsch and Roth. Creating motivating conditions in home settings for children's STEM learning using technology is guided by our interpretation of Vygotskian insight that "each higher form of behaviour enters the scene twice in its development-first as a collective form of behaviour, as an inter-psychological function, then as an intra-psychological function, as a certain way of behaving" (Vygotsky 1997, p.95). This movement from inter to intrapsychological plane is not a mere reflection of the outside world on an internal/ mental scheme but rather a dynamic and dialectical process that transforms a child's existing relationship with their social situation. This gives a very different reading to "screen time". We argue that concerns around children's screen time or use of digital technology assume that children's intrapsychological functioning is merely a reflection of what they engage in at the interpsychological (or social) level only. Children's agentic meaning-making and ability to create their social situation of development is discounted in these mechanistic understanding of children's engagement with digital tools.

Within Conceptual PlayWorlds play-based model, technology is theorised as a means that creates multiple and dynamic educational affordances for young children. As Valanides (2018) argued, what is critical in incorporating technology in formal and/ or informal educational settings is to transform the technical affordances of the actual technological tools to educational affordances for every child. However, research (Angeli and Valanides 2005; Valanides and Angeli 2008a, 2008b) has shown that distinguishing the technical functions of the technological tools from the educational affordances is not a given. Special attention is needed in order to shift the focus from the tool itself and focus on how the tool can be used as an amplifier that enriches, expands and transforms 
the child's learning experience in early childhood settings and/or in-home settings. With this lens in mind, we can better understand how the child and the digital technologies become mutually constituted through design. Therefore, the following sections illustrate how digital technology can be conceptualised and used in the home settings as a process of dynamic digital design, where the emphasis is given on what is new and innovative for the child's experience within the context of her/his family.

\section{Digital Technology in the Home Settings}

Arnott and Yelland (2020) have argued to view evolving digital technologies as "social, cultural and personal artefacts that can contribute to children's learning ecologies" (p.124). The apparent vacillation in parents about the use of digital technology in the home setting seems because of the bewitching and captivating power of the "virtual". As Valentine and Halloway (2002) highlighted,

"popular commentators often paint a picture of children as so immersed in on-line worlds that they turn away from the "real," becoming detached from off-line social and familial relationships and withdrawing from public outdoor space into on-line fantasy spaces. In these understandings, the "real" is represented as a fragile world under threat from the seductive lure of the "virtual" (Doel and Clarke 1999)" (Valentine and Halloway 2002, p. 304).

Even in the situations where parents consider the possibility of learning with/using technology, it is largely understood that technology can be used for learning about the social world but not directly related to concept learning. Parent's dominant understanding of technology use is that it is a tool for social networking and gathering information about the world (Engel et al. 2019, p.328) and often parents are not very clear on how it can be used as a tool for learning in their everyday world. The other understanding shown in the literature is that information communication technology (ICT) hinders children's learning. This fear draws on two interrelated lines of arguments. One, facet of it is the binary between real world and virtual (non-real) world and second is the developmental appropriateness of using technology. Picking up on these binaries Fleer (2019) has argued that boundary framing of digital tools and a clear binary between the digital and the real makes it difficult to conceptualise children's engagement with digital tools in early years. Marsh (2010) has argued that "children have been identified as gaining a range of benefits from the use of technology, such as technical and operational skills, knowledge and understanding of the world and subject-specific knowledge in areas such as literacy and mathematics", and this view has increasingly been presented in the digital literature (Marsh et al. 2005; Plowman et al. 2008). This highlights clarity in the design of learning or models that can create playful and engaging space for children using technology.

The focus in the Conceptual PlayWorld is not technology. Technology is in-service of children's inquiry to sustain and sometimes amplify their intentional projects. Drawing on Vygotsky's work in the cultural-historical conception of play, adults have an important and valued role that could sustain and introduce new excitement and drama to children's play but also model new ways of playing and engaging with their worlds. This is very true for digitally mediated conceptual PlayWorlds as well. It is increasingly important to understand how these digital devices create new cultural conditions for children's development. Moreover, from a Vygotskian position, learning of concepts is central to children's learning and development. Hedegaard 
concludes that "if we want to give children conceptual competence that is more oriented toward theoretical knowledge, we must make this part of their everyday practice. So, parents and educators should change the practice traditions that the children participate in to change the conceptual competences the child will acquire" (Hedegaard 2008, p. 275). Following Lindqvist (1995) when using the concept of playworld, it is meant "fictitious world (context) which children and adults come to share when they interpret and dramatize the theme in the classes" (Lindqvist 1995, p. 70). Unlike role-play where "everyone is free to make their own interpretations", in this paper, the term drama is also used to highlight that "everyone has to be part of a common fiction" (Lindqvist 1995, p. 37). In Conceptual PlayWorld, this emotional engagement of the child with the collective fiction is central to their concept learning.

This is one of the prime ideas guiding work at the Conceptual PlayLab at Monash University where the key focus is on orienting home/family practice traditions that can become motivating conditions for children's STEM concept formation. The practice of the home setting shown in this paper presents how digital and person mutually constitute motivating conditions for children's STEM concept formation in a Conceptual PlayWorld.

\section{Digital Tools in the Service of Conceptual PlayWorld}

A practice example of a pop-up Conceptual PlayWorld that creates motivating conditions for child's spatial concept formation has been shown here in the home setting of author 1 . We drew upon the everyday practices where digital tools gave new

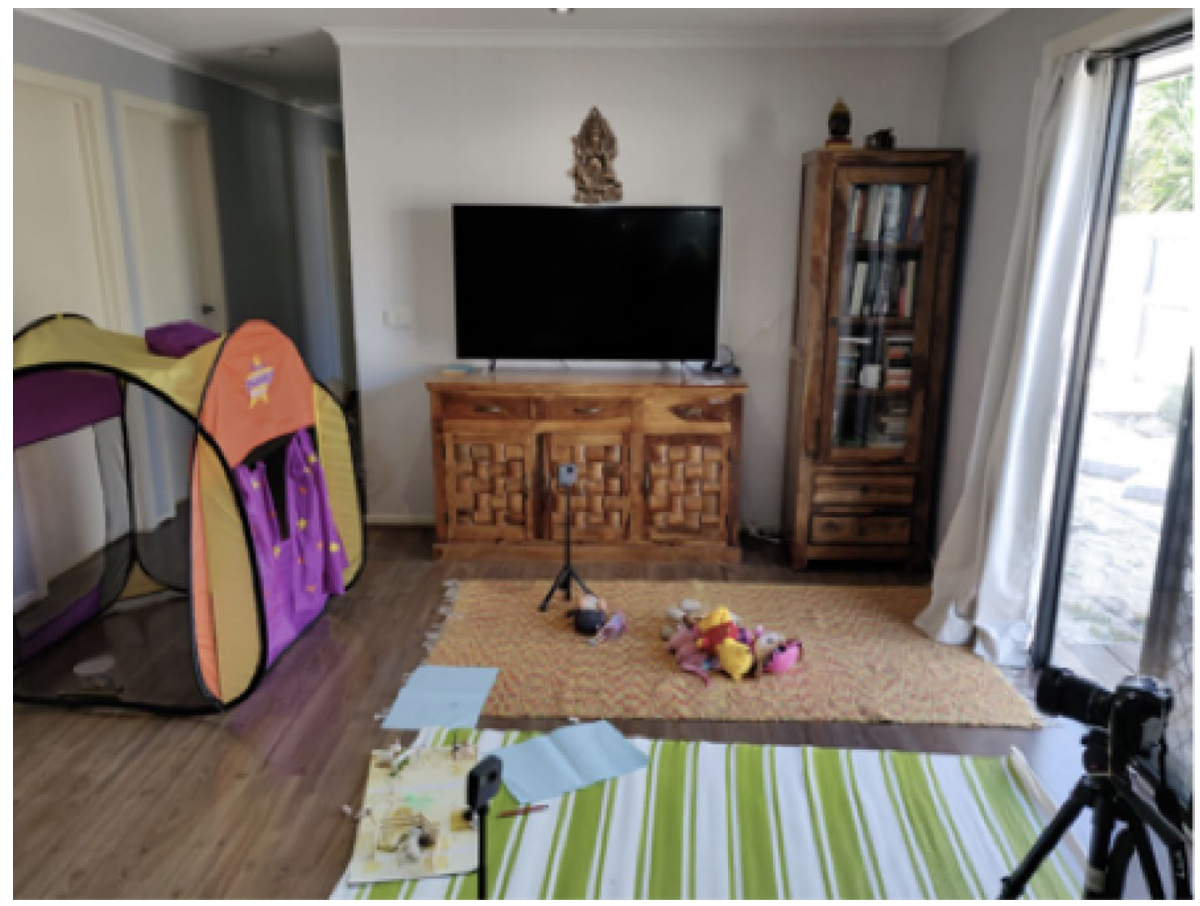

Fig. 1 Image showing position of the three cameras and the activity setting 
conditions in the family. Home setting of author 1 is used to illustrate the complexity of the theoretical problem that is the focus of this paper. The purpose in this practice example was to engage the child in spatial concepts of distance, location, direction and representation which can then further develop their capabilities in exploring, drawing and using maps. Two $360^{\circ}$ cameras and one standard video recording camera were used to understand the nature of interactions with the digital tools. All the cameras were mounted on a tripod. One camera was kept at the child's height and the other at an adult's height to best record both adult and child's perspectives. An image of the setting is shown below in Fig. 1. Fig. 2 shows some of the hand held puppets made with the child to do a Rosie's walk puppet show.

The child was very excited to see multiple gadgets and asked a number of questions to know more about how cameras record video. Parents used following five key characteristics of the Conceptual PlayWorld to create the imaginary play situation: (a) selecting a story that has drama and a complex plot that could engage children and introduces a problem situation regarding a STEM concept, (b) designing an imaginary play space to give children the opportunity to explore in different ways the STEM concepts, (c) entering and exiting the space creating collective STEM experiences, (d) planning inquiries, based on the story plot, in order to approach the STEM concept and (e) planning adult's role as he/she joins the imaginary space in order to interact with the children. The children's story book of Rosie's Walk by Pat Hutchins was used to develop this pop-up Conceptual PlayWorld. The home practice related to the Conceptual PlayWorld was followed over 3 days and in total $6 \mathrm{~h}$ of video data were recorded. Child's map drawing and her puppet colouring were also collected as part of the study. Two short videos, one by parents before they started to record the Conceptual PlayWorld and another after the Conceptual PlayWorld were recorded to understand the parent's perspective.

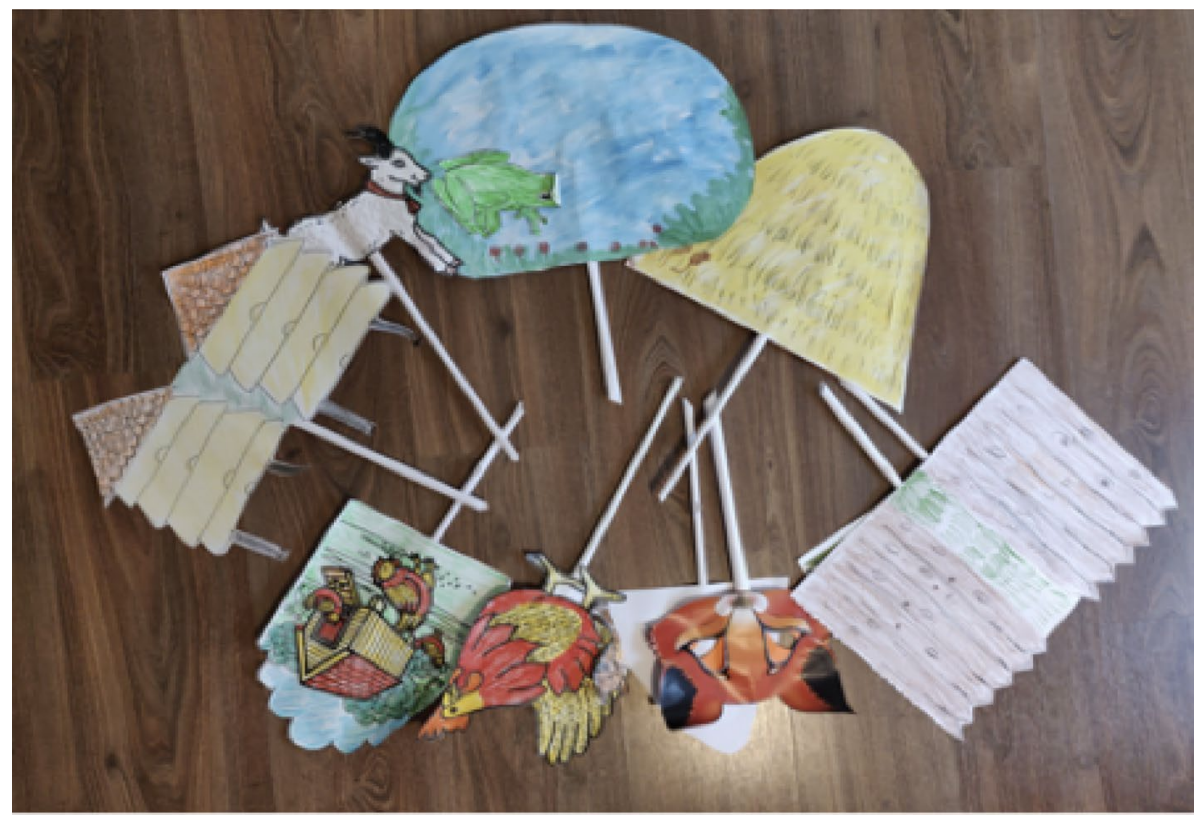

Fig. 2 Image of the various hand-held puppets made for the Rosie's Walk puppet show 


\section{Analysing Everyday Practices in Digital Conceptual PlayWorld}

Day 1: Preparation and planning for the pop-up Conceptual PlayWorld (mentioned now as pop-up CPW). Parents in this study used Fleer's Conceptual PlayWorld starters to orient themselves with the key characteristics of Conceptual PlayWorld. These starters offer a number of suggested routes to create a Conceptual PlayWorld; they also give access to digital resources that families can use to understand the STEM concepts better or get ideas to create play inquires. As the child had already read Rosie's Walk earlier, she was aware of the sequence of the story. Parents thus thought of presenting the story with a few props and in the puppet show tent of their daughter. This created extra excitement for her. The mother downloaded a few images of the story plots from the Internet. The child also searched with the mother and contributed in deciding the images. They also drew some of the images. Parents and child were engaged in colouring the various characters of the story. The child was very excited with the idea of puppet-making and colouring fox, ponds, hay and her favourite character Rosie, the Hen. The parents looked for some background music and sound (of fox and hen's) which could create an ambience for Rosie's Walk. They also decided to use the sound of the hand drum to highlight various transition points in the Conceptual PlayWorld, e.g. when the story ends or new activity of mapping starts. To support the child's inquiry and map drawing activity, the parents decided to show her a video from the Big History Project titled "Power of Ten" to problematise the idea of relative size and also to move the child from her two-dimensional mapping to a geospatial understanding. A street view map of the child's kinder and house from the Google Map was printed to facilitate conversation around mapping. The Google Earth and Google Map app were used on the iPad to show the difference between the geo-spatial view and two-dimensional view. Based on NCTM's (National Council of Teachers of Mathematics) guideline on spatial concept formation in early years, the following four aspects were emphasised in conversation with the child:

(a) Direction (which way?)

(b) Distance (how far?)

(c) Location (where?)

(d) Representation (what objects?)

Day 2: Implementing the pop-up CPW. The parents and child read the story of Rosie's Walk together; as they were reading, the mother who was sitting inside the puppet show tent showed movements of the characters using hand-held puppets. A few action sounds were played to highlight the drama in the story. The father was in the character of Fox, and the mother was playing Rosie's mother who was waiting for Rosie to have dinner together. As the story ended, the parents played a telephone ring sound from their iPad as if somebody is calling. The mother started to speak with somebody on the phone; the father was in the role of Rosie's friend Joey's father. A problem was introduced at this stage that Joey wants to come and play at Rosie's house but she does not know the way. There is also the danger of foxes still being around so she needs to be mindful of places where foxes can attack her. All this needs to be represented on the map so that Rosie's friend could reach her place without any trouble. When the problem situation was introduced, the child was unsure how to draw a map. She drew a sketch with very little detail just joining houses of Joey and Rosie (see Fig. 3). The only detail was a tree in front of Rosie's house. At this 


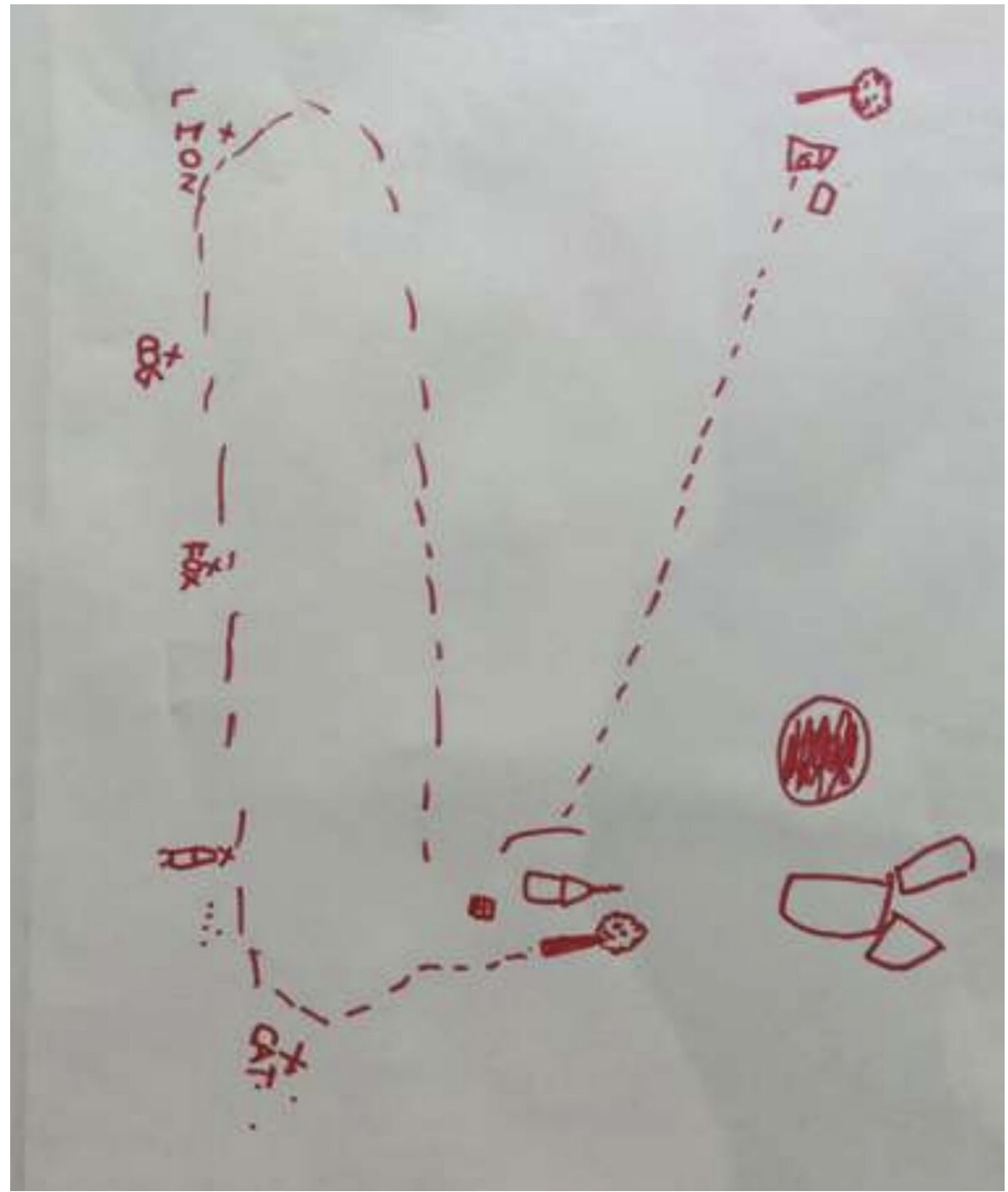

Fig. 3 Figure representing child's first effort of mapping of route from Rosie's and Joey's map

stage, the father came as Rosie's uncle who is a scientist and introduced the idea of looking for routes using Google maps(see. Fig. 4).

This was a very everyday experience for the child so she did not pay much attention to it. To sustain the drama and ensure further conceptual exploration, the scientist uncle asked Rosie does she know where these maps come from. The child mentioned satellites but she was not sure. She has heard that moon is a natural satellite. To explore the child's imagination further, the father asked the child if you have a big telescope and you are sitting on the moon, what will the Earth look like from there? The child got very excited and wondered if Earth would be very big and blue in colour 


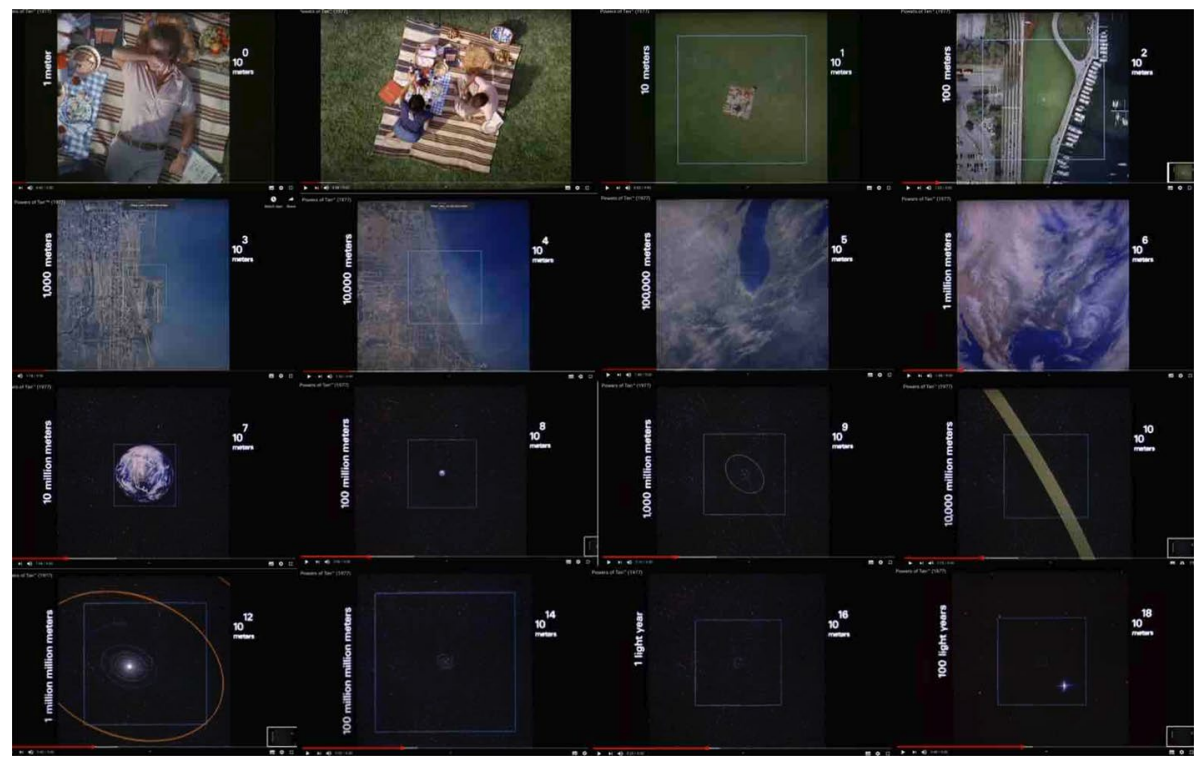

Fig. 4 Screenshot of the "Power of Ten" video showing relative scale

(like a globe). To further explore the concepts of direction, distance and location, the child was shown a 9-min video titled "Power of Ten" from the big history project that highlights the idea of relative size (screenshots of the video shown in Fig.4). The video starts with a family picnic in a park. The camera in the first instance shows a scene which is $1 \mathrm{~m}$ wide viewed from $1 \mathrm{~m}$ away, and then every $10 \mathrm{~s}$, it moves 10 times farther away from this family's scene and the field of view also becomes 10 times wider. It finally reaches to the view of earth from space and then finally to a point when the solar system shows a tiny and nearly insignificant dot in the space. This helped the child to consider how relative size of the object informs them being significant in a location. To sustain the child's further exploration and move from the two-dimensional representation of objects to plan-view the child was shown a planview of her own house. As the child was drawing different places, the parent asked how far is Joey's house from Rosie's house. This inquiry made the child think about how she can represent distance on her map. The conversation on the "Power of Ten" video then helped the child to think about scale.

The child was shown the Google route map from her house to the kinder. Various landmarks, locations, directions of turns and distance were highlighted to get her a sense of how digital maps work. This made her wonder if she could send a map like this to her friend Joey. The child re-drew her map and enacted as if she is telling direction according to the map for a while. The re-drawn map shown below (Fig. 5) had a lot more detail and a few extra characters beyond the story of Rosie's walk. The child drew a post-office next to which was a cat postman who could take map to Joey.(see. Fig. 5)

Day 3: The child designed her own Robot walk game. A day after the pop-up CPW after multiple improvisations, the child came to the father and asked him to participate in her new game called "Robot walk" (see Fig. 6). She also formulated 


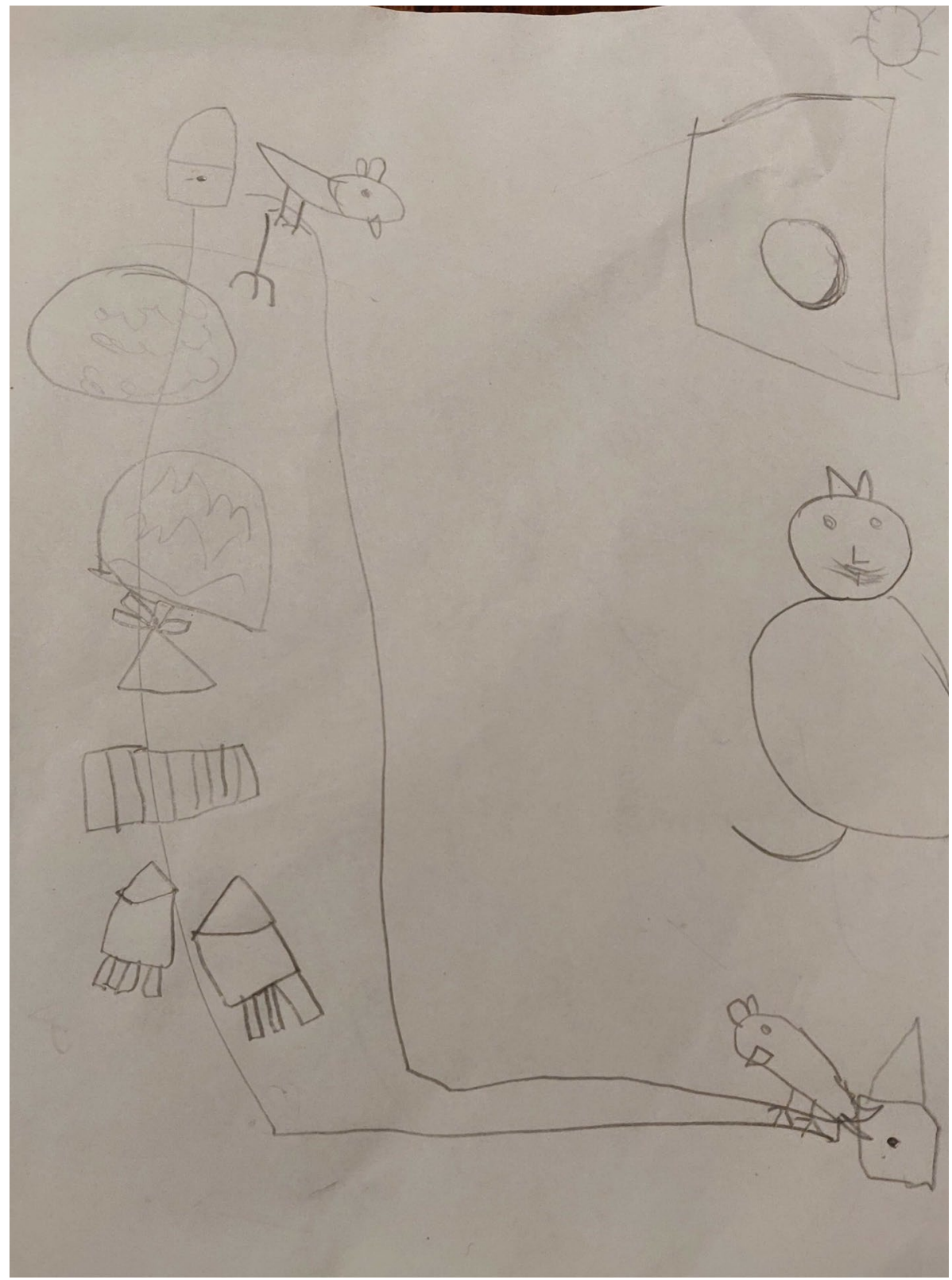

Fig. 5 Figure of the re-drawn map by the child

rules to play this game. Hiya, child's doll is sitting in her car that needed to be pulled. Hiya has her friends (other soft toys) in different rooms of the house. Child was acting as a robot who can take Hiya's car to different rooms. Father had to give direction so that Hiya could go to different rooms and meet her other friends. On instructions from her father, child use to move the doll's car left, right, forward or backward. This 


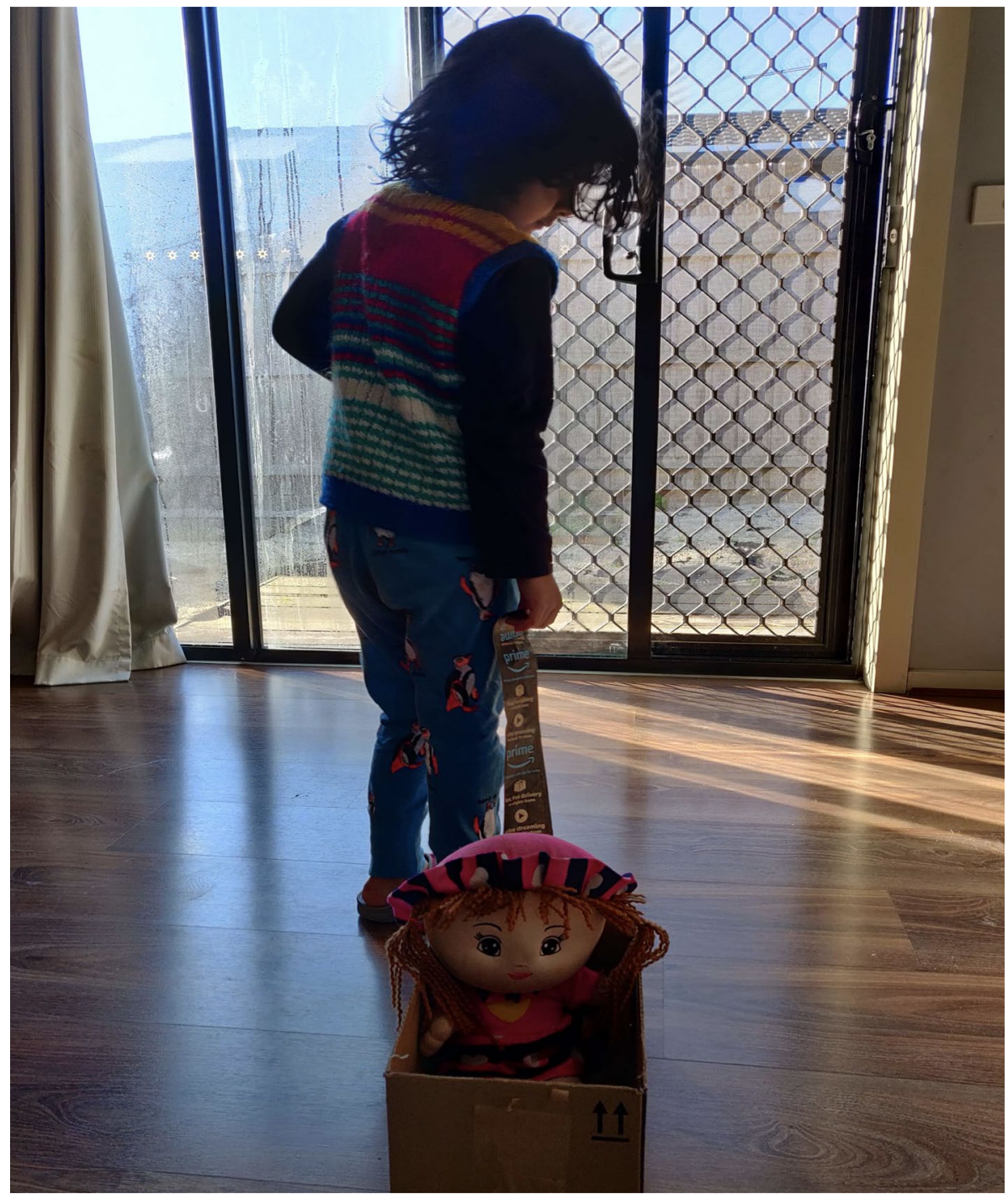

Fig. 6 Figure showing doll's car and robot walk played by the child

exploration took place for some time before she moved on to the next game. The child also came to her father and explored many other locations on google map (see Fig. 7) e.g. her playgrounds, distance between different countries.

\section{Solving Theoretical Problem}

The pop-up CPW showed that digital tools amplified and offered possibilities to further explore the STEM concepts in home settings. Two key aspects of practice especially became visible in the above pop-up CPW: 


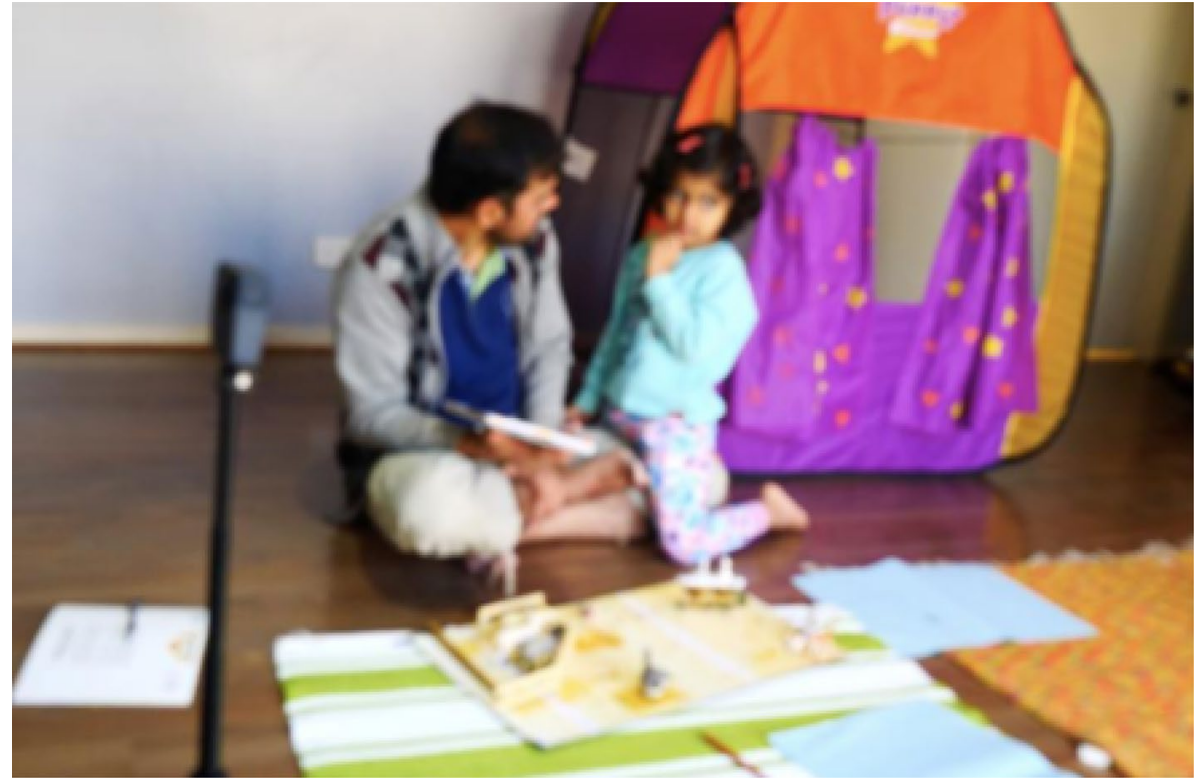

Fig. 7 Figure showing child asking her father to look for various locations on the map

\section{Relational engagement of child with digital artefacts}

The data shows that digital tools were part of the inextricable weave of the Conceptual PlayWorld settings. In the imaginary situation, the child was in the role of being Rosie, and a number of her own curiosities were expressed by the character. The digital tool created possibility for her further exploration.

The relational character of the digital tool was also visible as the child was making an effort to bring her own toys and make them part of the game. The Conceptual PlayWorld as a tertiary artefact supported children's imagination and also created an opportunity for the child to redevelop new roles for her dolls and toys with respect to technology as reflected in her own free play game "robot walk" where her father was helping the doll to find route to her friend. This does not reflect mere mimetic character of play but being agentic in creating a new meaning for her existing toys and dolls. This relational nature of technology highlights that there has been a boundary dissolution between technological and human world. A number of digital tools and apps used in the study were already part of child's everyday practice. This "everydayfication" of digital is all the more reason to move against the binary conceptualisation of digital and non-digital. In their everyday practice children use digital and other material artefacts together. The challenge is to use a design which incorporate and use these different artefacts to create motivating conditions for children's exploration and playfulness. The seamless integration of digital tools in children's play setting visible in the pop-up CPW supports Fleer (2017b) arguments of viewing digital tools as an auxiliary tool that facilitate or accentuate children's engagement in the activity setting.

\section{Spatial concept learning in the Conceptual PlayWorld}

Children's engagement in Conceptual PlayWorld also has a pedagogic purpose, and hence, these play settings were not free play space. The purpose of the activity was guided by developing spatial concepts in children. Introducing the problem scenario 
which is an important characteristic of Conceptual PlayWorld made the child more aware of the demands of the task. The problem of drawing a map for Rosie's friend presented a problem to the child. Her first drawing shown in Fig. 3 was mere joining of two points. A subsequent intervention using the digital tool where her father showed her Google maps of her street, route maps from her house to her kindergarten and then looking for locations on Google Earth made her think about Rosie's world and how it may look. When she redrew later (shown in Fig. 5), it has details like windmill, pond, lawn, etc. from the story of Rosie's walk. The child started to visualise map not merely as a location on the paper but she started to see a relational and a socio-emotional connection with it. The emotional drama and being in the character of Rosie helped the child to embody some of these relationships which were otherwise distant to her everyday thinking. Danby et al. (2016) have emphasised that "a sense of place is not just a physical location but having a social connection, a sense of belonging and display of affiliation on relationship to a location (Creswell 2004; Schegloff 1972; Shields 2006)" (p. 153). The digital experience of viewing Google maps and her street view on the map also gave an opportunity for the child to connect with her known locations differently. Even after the Conceptual PlayWorld was over she asked her father to search for her Kindergarten on the map a number of times (see Fig. 7). She was both amazed and excited by the possibility of seeing something from a distance using app like Google Earth. She also asked to search her house in India. Later she was interested in knowing if we could find a map to go from Australia to India to meet her grandparents. These examples also challenge the worries around "seductive lure of the "virtual" (Doel and Clarke 1999)". On the contrary, the child was seen to be bringing her everyday world to the virtual. The digital tools thus support children's meta-position with respect to their play. It facilitates the possibility to transcend and sometimes also transform their existing relationship with the concrete world. The study supports Fleer's (2017b) claims that the use of "digital devices... in playworlds, amplified the children's play in scientific ways, which in turn created new conditions for children's development" (p.303). These conditions brought to light and made conscious spatial concepts that gave new ways of thinking about the child's everyday world. But to understand how these concepts act as part of everyday life, a pedagogic lens is needed (see Table 1 further below).

Children's spatial concept "is the awareness of themselves in relation to the people and objects around them" (NCTM, 2000, p.98). As part of the Conceptual PlayWorld when the child started to draw the map the adult introduced exploratory questions e.g. which direction is Joey's house? How far is it? Is she going to take the same path which Rosie took on her walk? These exploratory questions helped to move thinking about the spatial relations in terms of other significant places around Rosie's house. Perspective-taking,

Table 1 Pedagogic everydayfication of digital tools for realising spatial concepts in home settings

\begin{tabular}{ll}
\hline Spatial concepts & Pedagogic focus \\
\hline Direction (which way?) & Moving the child from the body orientation to geo-spatial understanding \\
Distance (how far?) & Understanding scale and distance of various objects on the map \\
Location (where?) & Relative position of various objects on the map \\
Representation (what objects?) & Relative size of various objects in representation \\
\hline
\end{tabular}


navigating and renavigating and detailing the scene using her imagination was visible as the father (researcher) showed different kinds of maps to the child. Digital technology helped to see the relationship between two-dimensional and a plan-view.

It was through this process of digitally amplified practice with its orientation to spatial knowledge that we can see how the child becomes aware of relative position, distance and direction. An emerging understanding of representation is also visible from Fig. 5. The child moved from writing name of the places to representations of places and animals. In particular, the example shown here points towards a Vygotskian focus on theoretical knowledge or scientific concepts for teaching-learning. The scientific concept here underpins practices that could guide development of higher mental functions in children and particularly learning of STEM concepts.

\section{Conceptual PlayWorld as a Model/Design for Digital Engagement in Early Years}

This brief intervention highlights a few key possibilities for children's use of digital technology especially in challenging COVID-19 world when their access to early care centres is restricted. Children's story and role play created a "collective fiction" that informed the child's participation and also concept learning in the home setting. The relational nature of technology is important because children's commitment to the collective fiction ensured their participation in the collective imagination, and hence, there is no moment-to-moment negotiation but an organic movement between the various activities as adult-child moves between various activities. The digital experience created the possibility for the child to imagine beyond her immediate material world. There was also a possibility for her to collectively imagine with the parents about their geo-spatial location. Taken together, this was an example of an "everydayfication" of digital technology in the home setting realised through the collective fiction and imagining within the Conceptual PlayWorld. Wartofsky (1979) in his book "Models: Representations and the Scientific Understanding" described models in a "special sense... when one wants to talk about inventing the future". Seeing models as modes of action they were seen as "embodiment of creative human purpose" (p.145). They were not seen as instruments to be used but themselves modes of action" (p.142) Conceptual PlayWorld in this paper helps to offer a "shared script" that does not demand repeated negotiation but offer a "mode of action" where children are co-participant in creation of their social situation of development. The paper makes a case for "model or learning design" for ensuring children's engagement with digital technology in post-COVID situations instead of offering a mosaic approach or a collection of tools and apps. It can be argued that Conceptual PlayWorlds acted as a tertiary artefact that offers a digital design to children's play settings. Digital tools as seen in this study amplified the children's play and supported their playful explorations.

\section{Conclusions}

The purpose of this paper was to discuss a pressing theoretical problem of how person and digital technologies become mutually constituted in the emergent context of COVID19. This problem has emerged through the unprecedented use of digital tools in family homes. Out of necessity, we are seeing a plethora of images of families sitting at what was once the preserve of the dinner table, sharing devices and engaging in work from 
home, participating in remote playgroups and playdates, engaged in remote school learning from home, and homework of a different order. Within this dynamic home space, we have seen how new institutional demands on family homes for engaging remotely with the school, preschool and workplace have changed the institutional structure and practices of the family-visually, organisationally and importantly for the focus of this paper, psychologically. The device and the need for the device have created new ways of being at home. It was in this context that we introduced a model of a Conceptual PlayWorlds for better understanding this dynamic and changing context so that we could analyse how person and digital technologies become mutually constituted.

By deliberately bringing into the family home of the first author, a digitally designed Conceptual PlayWorld, we undertook to theorise the problem dialectically. Theorised from a cultural-historical perspective we went beyond binaries of digital and non-digital for conceptualising the problem as shown in the literature as screen time. Digital design from a cultural-historical perspective gave us entre into reframing the new practice tradition of using digital tools as a synthesis of person and environment. We found through 3 days of following how the family engaged in everyday practices of a Conceptual PlayWorld that person and digital were always in relations with each other. We theorised this as an "everydayfication" of digital technology in the home setting. Key to this theorisation was how family and digital technologies became mutually constituted through the collective fiction and imagining within the Conceptual PlayWorld. It was not possible to separate out screen time because both what was on the screen and what was realised in play practice were always in motion and always dialectically related to each other. The figures in this paper show this relation quite explicitly.

In going beyond a binary, the results of our theorisation showcase the need for organisations which give guidelines on screen time to foreground in their advice the dynamic nature of what is constituted between person and the digital technology. Rather than conceptualised as digital consumerism, organisations can broaden their net to bring in the person and what is amplified in a bidirectional way. Rather than moral panic about screen time or digital consumerism as framing central arguments as has also emerged within the literature, we suggest that future studies and conceptualisations of digital tools be thought about as "everydayfication" of digital technology in the home setting. The lens moves from the digital tool and towards how person and digital technologies become mutually constituted as part of the changing practice tradition of homes in times of COVID-19.

Acknowledgments We would like to acknowledge the research assistance of Sue March and Abbey McLean.

Funding Funds from the Australian Research Council [FL180100161] to support the research reported in this paper.

\section{References}

Arnott, L., \& Yelland, N. (2020). Multimodal lifeworlds: Pedagogies for play inquiries and explorations. Journal of Early Childhood Education Research, 9(1), 124-146.

Angeli, C., \& Valanides, N. (2005). Pre-service teachers as ICT designers: An instructional design model based on an expanded view of pedagogical content knowledge. Journal of ComputerAssisted Learning, 21(4), 292-302. 
Brown, J. S., Collins, A., \& Duguid, P. (1989). Situated cognition and the culture of learning. Educational Researcher, 18(1), 32-42.

Cole, M. (2019). Re-covering the Idea of a Tertiary Artifact. In A. Edwards, M. Fleer, \& L. Bøttcher (Eds.), Cultural-Historical Approaches to Studying Learning and Development: Societal, Institutional and Personal Perspectives (pp. 303-321). Singapore: Springer Singapore.

Danby, S., Davidson, C., Ekberg, S., Breathnach, H., \& Thorpe, K. (2016). 'Let's see if you can see me': Making connections with Google EarthTM in a preschool classroom. Children's Geographies, 14(2), 141-157.

Derry, J. (2007). Epistemology and conceptual resources for the development of learning technologies. Journal of Computer Assisted Learning, 23(6), 503-510.

Doel, M., \& Clarke, D. (1999). Virtual worlds: Simulation, suppletion, s (ed) uction, and simulacra. In M. Crang, P. Crang, \& J. May (Eds.), Virtual geographies: Bodies, space, and relations (pp. 261-283). London: Routledge.

Engel, A., Fauré, J., Membrive, A., Merino, I., \& Coll, C. (2019). The influence of parents in the discursive construction of technology-mediated learning experiences. Mind, Culture, and Activity, 26(4), 323-335.

Fleer, M. (2019). Digitally amplified practices: Beyond binaries and towards a profile of multiple digital coadjuvants. Mind, Culture, and Activity, 26(3), 207-220.

Fleer, M. (2017a). Digital role-play: The changing conditions of children's play in preschool settings. Mind, Culture, and Activity, 24(1), 3-17.

Fleer, M. (2017b). Digital playworlds in an Australia context. In T. Bruce, M. Bredikyte, \& P. Hakkarainen (Eds.), Routledge handbook of play in early childhood (pp. 289-304). Abingdon, UK: Routledge Press, Taylor and Francis.

Gillespie, A., \& Zittoun, T. (2010). Using resources: conceptualizing the mediation and reflective use of tools and signs. Culture and Psychology, 16(1), 37-62.

Hedegaard, M., \& Fleer, M. (2008). Studying children: a cultural-historical approach. Maidenhead, England; New York: Open University Press.

Hedegaard, M., \& Fleer, M. (2013). Play, learning and children's development. Everyday life in families and transition to school. New York: Cambridge University Press.

Johnson-Laird, P. N. (1983). Mental models: Towards a cognitive science of language, inference, and consciousness (No. 6). Cambridge, MA: Harvard University Press.

Lantolf, J. P., \& Appel, G. (Eds.). (1994). Vygotskian approaches to second language research. Norwood: Ablex Publishing Corporation.

Lindqvist, G. (1995). The aesthetics of play: A didactic study of play and culture in preschools. Doctoral thesis-Uppsala University.

National Council of Teachers of Mathematics. (2000). Principles and standards for school mathematics. Reston, VA: National Council of Teachers of Mathematics.

Marsh, J. (2010). young children's play in online virtual worlds. Journal of Early Childhood Research, $8(1), 23-39$.

Marsh, J., Brooks, G., Hughes, J., Ritchie, L. \& Roberts, S. (2005) Digital Beginnings: Young Children's Use of Popular Culture, Media and New Technologies. Sheffield: University of Sheffield. Available online at http://www.digitalbeginings.shef.ac.uk

Plowman, L., McPake, J., \& Stephen, C. (2008). Just picking it up? Young children learning with technology at home. Cambridge Journal of Education, 38(3), 303-319.

Ravanis, K. (2000). La construction de la connaissance physique à l'âge préscolaire : recherches sur les interventions et les interactions didactiques. Aster, 31, 71-94.

Ravanis, K. (2005). Les Sciences Physiques à l'école maternelle: éléments théoriques d'un cadre sociocognitif pour la construction des connaissances et/ou le développement des activités didactiques. International Review of Education, 51(2/3), 201-218.

Schegloff, E. A. (1972). Notes on a Conversational Practice: Formulating Place. In D. Sudnow (Ed.), Studies in Social Interaction (pp. 75-120). New York: The Free Press.

Shields, R. (2006). Knowing space. Review of Theory, Culture \& Society, 23(2-3), 147-149.

Valanides, N., \& Angeli, C. (2008a). Professional development for computer-enhanced learning: A case study with science teachers. Research in Science and Technological Education, 26(1), 3-12.

Valanides, N., \& Angeli, C. (2008b). Learning and teaching about scientific models with a computer modeling tool. Computers in Human Behavior, 24(2), 220-233.

Valanides, N. (2018). Technological tools: From technical affordances to educational affordances. Problems of Education in the 21st Century, 76(2), 116.

Valentine, G., \& Holloway, S. L. (2002). Cyberkids? Exploring children's identities and social networks in on-line and off-line worlds. Annals of the Association of American Geographers, 92(2), 302-319. 
Vygotsky, L. S. (1997). The collected works of L.S. Vygotsky: Problems of the theory and history of psychology (R. van der Veer, Trans. R. W. Rieber and J. Wollock Eds., Vol. 3). New York, NY: Kluwer Academic and Plenum Publishers.

Wartofsky, M. W. (1979). Models : Representation and the scientific understanding. Dordrecht, Holland ; Boston: D. Reidel Pub. Co.

Wertsch, J. V. (2007). Mediation. In H. Daniels, M. Cole, \& J. V. Wertsch (Eds.), The Cambridge companion to Vygotsky (pp. 178-192). New York: Cambridge University Press.

Publisher's Note Springer Nature remains neutral with regard to jurisdictional claims in published maps and institutional affiliations. 